$$
x_{f}^{\prime}=\int_{0}^{t_{f}}\left[2 \int_{0}^{t} a(t) \mathrm{d} t-\int_{0}^{t_{f}} a(t) \mathrm{d} t\right] \mathrm{d} t+x_{i} .
$$

It is apparent from (5) that, with an $F(t)$, solutions of (1) will not in general have the property of path reversibility ; for the reversed motion does not, after time $t_{f}$, give $x_{f}^{\prime}=x_{i}$. (A sinusoidal force, $F=b \sin \omega t$, provides a simple example of an $F(t)$.) In the special case that $a(t)$ is a constant, (5) reduces to $x_{f}^{\prime}=x_{i}$; and, as noted, we do have path reversibility when (1) is solved with an $F^{\prime}(x)$. It appears that motions under various kinds of physical forces must be individually examined for their reversibility properties.

Department of Physics,

Michigan State University,

East Lansing, Michigan.

1 Popper, K. R., Nature, 177, 538 (1956). 2 Morpurgo, G., Radicati, L. A., and Touschek, B. F., Nuovo Cimento,
12, 677 (1954).

Mr. R. SchleGEL's definition of temporal reversibility, which he credits to Morpurgo, Radicati and Touschek, is clearly too narrow. It has to be extended, so as to cover a (conservative) physical system rather than one single particle.

Take the case of a planetary system : if we reverse the velocity of one of the planets, at the time $t_{f}$ and at the position $x_{f}$, the planet will clearly not reverse its path precisely: upon reaching, at the time $t_{f}+\Delta t$, the position $x_{f}+\Delta x$, which we may assume to be very nearly the same as the one which it occupied at $t_{f}-\Delta t$, the planet will not be acted upon by the same force which acted upon it at the time $t_{f}-\Delta t$. If, however, we reverse the motions of all the planets in the system, then the force will be the same; the system is reversible.

This shows that the problem of reversibility or irreversibility arises only with respect to a (conservative) physical system. It is obvious that, in a classical model, the motion of one planet or of one particle cannot be reversed.

$\mathrm{Mr}$. Schlegel proposes to consider the reversibility of the motion of one particle under the action of a time-dependent force. There are, clearly, two cases to be considered. In the first case, the temporal changes of the force in question are explicable (that is, deducible, or predictable) in terms of the changes within the system ; in classical mechanics, in terms of the changing positions of the various particles. Classical mechanics is reversible in the sense that if the velocity vectors of all particles are reversed, the temporal and positional changes of the forces (but not their directions, of course) are also reversed, and all the particles return on the paths on which they came. In the second case, the changes of the force in question are not explicable in terms of the changes in the system. In this case, the problem of reversibility, and of the 'arrow of time', does not arise: irreversibility is trivial, except under the assumption that the temporal changes of the force are also reversed.

Mr. Schlegel operates with a changing force the changes of which he does not reverse. He therefore obtains the obvious result : the motion of his particle will not in general be reversible.

Concerning Mr. Schlegel's comments on my own communication, I wish to make four remarks.

(1) I have found since that nearly half a century ago, Einstein used a somewhat similar argument ${ }^{1}$.
Had I known this, I would not have written my communication.

(2) The difference between Einstein's argument and mine is that I have pointed out $(a)$ that the generators would have to be coherent, and $(b)$ that the demand that the coherence of the generators should be explained, and the explanation incorporated into the model, must either be given up (which means accepting the process as irreversible) or it will lead to an infinite regress. Mr. Schlegel mentions coherence, but he does not appear to have fully appreciated my admittedly very brief sketch of the way in which we are led to an infinite regress, if we do not wish to accept the coherence of the generators as an ultimate and inexplicable conspiracy of causally unrelated conspirators.

(3) As to dissipating processes, there are two kinds of dissipation which do not always seem to be clearly distinguished. Dissipation in the form of increasing disorder (entropy increase) is one of them, and dissipation by expansion without increase of disorder is the other. For an increase of disorder, walls of some kind are essential : a sufficiently thin gas expanding in a 'vessel without walls' (that is, the universe) does not increase its disorder. In the absence of walls, collisions become rapidly extremely improbable, and the degree of order of the system begins to increase in time, in the sense that the fastest particles will all be found near the periphery of the expanding gas ball and the slowest particles near its centre. If this development is prevented by the insertion of 'walls' of some kind (the 'walls' may be gravitational fields preventing the escape of particles) then the disorder will increase.

(4) My earlier brief communication was merely an attempt to point out the untenability of the widespread, though surely not universal, belief that the 'arrow of time' is closely connected with, or dependent upon, the law that disorder (entropy) tends to increase. Beyond this, I did not attempt to cover the subject.

University of London.

K. R. POPPER

2 Einstein, A., Phys. Z., 10, 821 (1909).

\section{Application of Impedance Measurements to Corrosion Phenomena}

Is connexion with a recent communication ${ }^{1}$ it should be pointed out that the application of impedance measurements to corrosion phenomena is not new. Extensive measurements of the resistance and capacity of painted steel specimens have been carried out for a number of years ${ }^{2}$ and have proved most useful in studying the breakdown of paint films.

In the course of this work, the possibility was raised of extending the method to other thin films such as anodic oxide films. The present work ${ }^{1}$ demonstrates the usefulness of the method in the study of phe. nomena within oxide films formed during corrosion.

Motallurgy Division,

J. N. WANKLYN

Atomic Energy Research Establishment, Harwell, Didcot, Berks.

\section{M. Brasher \\ F. WORMWELL}

Chemical Research Laboratory, Teddington, Middlesex.

1 Wanklyn, J. N., Nature, 177, 849 (1956).

2 Wormwell, F., and Brasher, D. M., Nature, 159, 678 (1947) ; see also "Chemistry Research, 1947", 11 (H.M.S.O., London, 1949); 